\title{
SCIENCE@LERT
}

\section{Landscaper seeks remunerative position}

Woodford-Richens K, Williamson J, Bevan S, et al. Allelic loss at SMAD4 in polyps from juvenile polyposis patients and use of fluorescence in situ hybridization to demonstrate clonal origin of the epithelium. Cancer Res 2000;60:2477-82.

\section{Abstract}

Juvenile polyposis syndrome (JPS) is an inherited genetic defect resulting in production of multiple hamartomas, some of which subsequently develop into carcinomas. About $30 \%$ of these patients are known to have a heterozygotic defect in the SMAD4 gene that codes for a mediator of transforming growth factor beta signalling. The loss of one of the two normal SMAD4 alleles is not thought to be sufficient to induce hamartomas but requires the additional loss of the residual normal allele as a secondary event. In patients with JPS, the hamartomas were thought to result from loss of the second normal allele in stem cells that produced stromal cells and equally importantly, that the overlying epithelium continued to have one copy of the normal allele. On this basis, the subsequent development of carcinoma of the epithelium was considered to be due to the epithelial cells being positioned in a highly abnormal microenvironment ("soil", hence landscaper theory). In this paper, Woodford-Richens et al used fluorescence in situ hybridisation (FISH) directed against the SMAD4 gene to probe individual cells of the polyps to determine which had lost both copies of SMAD4. They found that cells of the stroma and epithelium, but not the inflammatory infiltrate, had lost both alleles. A complicated theory involving "cross talk" between a normal overlying epithelium and an abnormal stroma does, therefore, not have to be invoked to explain why the epithelial cells subsequently undergo malignant transformation. In addition, the finding of identical secondary genetic defects in both the epithelium and stroma of the hamartomas suggests that they originate from the same stem cells and not from distinct lineages as previously thought.

\section{Comment}

In 1998, Kinzlker and Vogelstein proposed a hypothesis that expanded the limited data available from known specific genetic defects into a general mechanistic process underlying malignant transformation of the gastrointestinal tract. ${ }^{1}$ In this model, classic tumour suppressor genes such as $A P C$ function as "gatekeepers", preventing, in the case of $A P C$, the translocation of $\beta$-catenin to the nucleus where it complexes with Tcf- 4 and induces, inter alia, expression of c-myc, with ensuing increased cell proliferation and selection. Secondly, DNA repair proteins such as $M L H 1$ and $M S H 2$ act as "caretakers" of the genome, correcting mismatches in potentially important genes and thus preventing their inappropriate expression. Finally, in a series of diseases as disparate as inherited polyposis syndromes such as juvenile polyposis syndrome (JPS), and acquired conditions such as ulcerative colitis, changes in the stromal component of the lesions-the clonal stromal component of the hamartomas of JPS and the inflammatory infiltrate in ulcerative colitis - result in an altered terrain for epithelial cell growth which increases cancer susceptibility (the "landscaper" hypothesis or effect). In this model, it is envisaged that mutations in the stroma of the hamartomas in some way modulate epithelial cell proliferation through epithelial:mesenchymal interactions or epithelial damage (fig 1) while the cocktail of cytokines secreted by the inflammatory infiltrate have similar effects on the colonic epithelium in ulcerative colitis. The detailed molecular processes underlying this epithelial:mesenchymal "cross talk" remain to be elucidated although several examples have now been demonstrated. For example, large

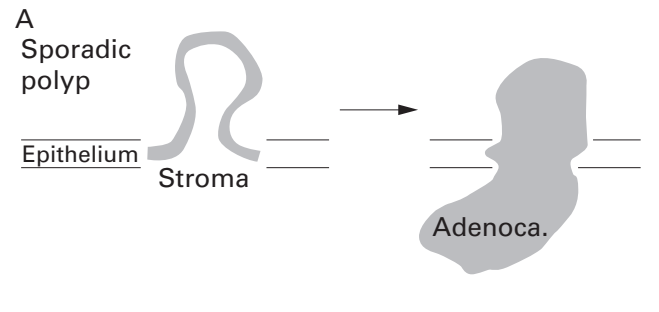

B

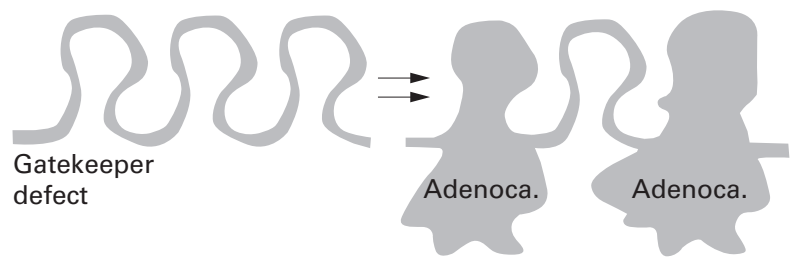

C

Kinzlker and Vogelsteins'

proposed landscaper

defect

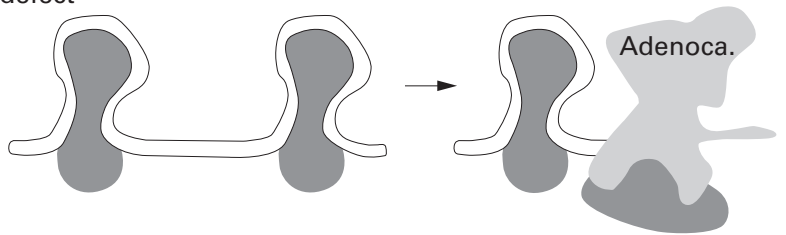

Figure 1 Schematic representation of colonic mucosa, divided into stroma and overlying epithelium. Regions with functionally relevant genetic defects are shown in light and dark grey. Adenoca., adenocarcinoma. (A) Sporadic colonic tumours acquire initial functional genetic defects in specific regions of the epithelium, causing dysplastic polyps that may progress to carcinoma. (B) In patients with conditions such as familial adenomatous polyposis, the whole of the epithelium is affected by a defect in a tumour suppressor gene, resulting in the development of multiple dysplastic polyps. Some of these polyps will subsequently progress to carcinoma. A defect in a tumour suppressor gene resulting in multiple polyps can be considered a loss of control of gatekeeper function. (C) Patients with juvenile polyposis syndrome (FPS) initially develop hamartomas that were thought to have functional genetic defects (that is, loss of the second functional allele of SMAD4) only affecting the stroma. The overlying epithelium was considered to be still under

functional genetic control of the remaining normal SMAD4 allele. According to the landscaper hypothesis, this abnormality of the stroma subsequently induced the overlying epithelium to progress to carcinoma. The recent paper

by Woodford-Richens et al casts doubt on the relevance of this model in patients with FPS as they have identical genetic defects affecting the overlying epithelium. 
fold gastritis is a premalignant gastric condition that involves Helicobacter pylori colonisation causing an increase in cytokine (interleukin $1 \beta$ ) production by the inflammatory infiltrate. This in turn stimulates hepatocyte growth factor production and release by mesenchymal cells, resulting in increased epithelial proliferation due to the released hepatocyte growth factor binding to c-met receptors on epithelial cells. ${ }^{2}$ In conditions such as these, a general stimulation of proliferation may allow subpopulations of cells with genetic defects to expand. Alternatively, the constituents of the inflammatory cocktail, such as nitric oxide, free radicals, and the cytokines themselves, may result in direct genetic injury. ${ }^{34}$ In addition, modulation of cytokine and growth factor production might influence cell growth and cell:cell interactions via mechanisms such as E-cadherin downregulation and $\beta$-catenin signalling.

While the jobs of the "caretaker" and "gatekeeper" appear safe, at least for the moment, a recent paper by WoodfordRichens and colleagues" has made the position of the "landscaper" if not redundant at least tenuous. Epithelial malignancies are increased in incidence in both JPS and ulcerative colitis. The "landscaper" effect was coined to explain the apparent paradox of a stromal lesion-the hamartomas of JPS - predisposing to an epithelial malignancy: the abnormal stromal environment affects the development and growth of epithelial cells. JPS is one of several hamartomatous polyposis syndromes which include PeutzJeghers syndrome and Cowden's syndrome, all of which show multiple polyps in the gastrointestinal mucosa, with cystically dilated glands and a cellular stroma composed of smooth muscle, fibroblasts, and myofibroblasts. The spectrum of organ specific malignancies in these syndromes is wide, with Peutz-Jeghers syndrome predisposing to breast, cervix, and gastrointestinal cancers, Cowden's syndrome to thyroid and gastrointestinal tumours, and JPS to carcinomas occurring in the intestine and stomach.

The molecular pathology of these lesions is now being studied: in Peutz-Jeghers syndrome, the polyp epithelium shows clonal allelic loss at the LKB1/STKII locus (encoding a serine-threonine kinase) on chromosome $19 \mathrm{p} 13.4,{ }^{6}$ and carcinomas arising in Peutz-Jeghers syndrome show loss of this wild-type allele, strongly favouring evolution from hamartomas to carcinoma. ${ }^{78}$ Moreover, hamartomas, and both adenomas and carcinomas in Cowden's syndrome, show loss of heterozygosity at the PTEN/MMAC1 locus on chromosome $10 \mathrm{q} 23.3,{ }^{8}$ indicating that further loss of the wild-type allele induces growth of the hamartoma with occasional progression to carcinoma, in the classical tumour suppressor gene mode. ${ }^{9}$

In JPS, germline mutations in the SMAD4, or DPC4 gene on chromosome 18q21.1, are seen in a proportion of cases. Most SMAD4 mutations produce a truncated protein that inactivates its function as a cytoplasmic mediator in the transforming growth factor $\beta$ signalling pathway. SMAD4 appears to act as a classical tumour suppressor (gene) in the colon ${ }^{10}{ }^{11}$ and pancreas, ${ }^{12}$ and importantly, a high incidence of colorectal cancer has been reported in a large JPS kindred, linked to 18q21.1, with mutation in SMAD4, further suggesting that SMAD4 acts as a tumour suppressor gene in JPS..$^{13}{ }^{14}$

Using microsatellites, Woodfood-Richens and colleagues ${ }^{5}$ have demonstrated that JPS polyps containing a constitutional mutation often lose the wild-type SMAD4 allele, strongly suggesting that somatic loss of this allele is the first somatic mutation, inducing the growth of the hamartoma. Thus hamartomas in JPS resemble colonic adenomas, rather than primarily stromal lesions as previously thought. ${ }^{1516}$ Moreover, FISH showed loss of SMAD4, not only in epithelial cells but also in stromal fibroblasts and pericryptal myofibroblasts, but not in lymphocytes. ${ }^{16}$
These findings indicate that the epithelium in JPS polyps is clonal and somewhat surprisingly, this clonality is shared by a component of the stroma, suggesting that the precursors of these lesions are laid down very early in development, before epithelial:mesenchymal differentiation in the intestinal anlage, with later clonal expansion. Alternatively, the mutation could occur later in life as a stem cell with plasticity of a greater degree than is usually considered. ${ }^{17}$ Certainly other neoplasms with multiple lineages, such as hamartomas in tuberous sclerosis ${ }^{18}$ and mixed mullerian tumours which contain both epithelial and mesenchymal elements, appear clonal. ${ }^{19}$

This brings the epithelium into sharp focus in the formation of the hamartoma and its progression to carcinoma. Thus in JPS, and also possibly in other hamartomatous lesions such as Peutz-Jeghers syndrome and Cowden's syndrome, the development of epithelial malignancy is likely to be due to direct progression of the epithelial component. Therefore, although the previous suggestion of complex epithelial:mesenchymal interactions remains a possibility in the causality of malignant development of JPS, invocation of Occam's razor dictates that the most "straightforward" mechanism (involving the minimum number of assumptions) should be considered to be the most likely. There is no need therefore to incriminate the "landscaper" hypothesis: in these lesions, job flexibility or retraining to the "gatekeeper" career pathway seems more appropriate. Whether or not openings are available for landscapers in other terrains, such as the inflamed colon of patients with ulcerative colitis, remains to be seen.

R J PLAYFORD

Department of Gastroenterology, Imperial College School of Medicine, Hammersmith Hospital, Ducane Road, London W12 ONN, UK r.playford@ic.ac.uk

1 Kinzlker KW, Vogelstein B. Landscaping the cancer terrain. Science 1998;280:1036-7.

2 Yasunaga Y, Shinomura Y, Kanayama S, et al. Increased production of interleukin $1 \mathrm{~b}$ and hepatocyte growth factors may contribute to foveolar hyperplasia in enlarged fold gastritis. Gut 1996;39:787-94.

3 Lazutka JR. Genetic toxicity of cytokines. Mutat Res 1996;361:95-105.

4 Tamir S, deRojas-Walker T, Wishnok JS, et al. DNA damage and genotoxicity by nitric oxide. Methods Enzymol 1996:269:230-43.

5 Woodford-Richens K, Williamson J, Bevan S, et al. Allelic loss at SMAD4 in polyps from juvenile polyposis patients and use of fluorescence in situ polyps from juvenile polyposis patients and use of fluorescence in situ
hybridization to demonstrate clonal origin of the epithelium. Cancer Res 2000;60:2477-82.

6 Hemminski A, et al. Localisation of a susceptaibility locus for Peutz-Jeghers syndrome to $19 \mathrm{p}$ using compartative genomic hybridisation and targeted linkage analysis. Nat Genet 1997;15:87-90

7 Gruber S, Entius MM, Petersen GM, et al. Pathogenesis of adenocarcinoma in Peutz-Jeghers syndrome. Cancer Res 1998;58:5267-70.

8 Wang Z, Ellis I, Zauber P, et al. Allelic imbalance at the LKB1 (STK11) locus in tumours from patients with Peutz-Jeghers syndrome provides evidence for a hamartome-(adenoma)-carcinoma sequence. $\mathcal{F}$ Pathol 1999; 188:9-13.

9 Marsh DJ, Dahia PL, Coulon V, et al. Allelic imbalance, including deletion of PTEN/MMACI, at the Cowden disease locus on 10q22-23, in hamartomas from patients with Cowden syndrome and germline PTEN mutation. Genes Chrom Cancer 1998;21:61-9.

10 Tagaki Y, Kohmura H, Futamura M, et al. Somatic alterations of the DPC4 gene in human colorectal cancers in vivo. Gastroenterology 1996;111:136972 .

11 MacGrogen D, Pegram M, Slamon D, et al. Comparative mutational analysis of DPC4 (Smad4) in prostatic and colorectal carcinomas. Oncogene 1997;15:111-14.

12 Moskaluk CA, Roth S, Ringold JC, et al. Genomic sequencing of DPC4 in the analysis of familial pancreatic carcinoma. Diagn Mol Pathol 1997;6:8590 .

13 Howe JR, Roth S, Ringold JC, et al. Mutations in the SMAD4/DPC4 gene in juvenile polyposis. Science 1998;280:1086-8.

14 Houlston R, Bevan S, Williams A, et al. Mutations in DPC4 (SMAD4) cause Peutz-Jeghers syndrome but only account for a minority of cases. Hum Mol Genet 1998;7:1907-12.

15 Veale A, McColl I, Bussey H, et al. Juvenile polyposis coli. 7 Med Genet 1966;3:16.

16 Jacoby RF, Schlack S, Cole CE, et al. A juvenile polyposis tumour suppressor locus at $10 \mathrm{q} 22$ is deleted from nonepithelial cells in lamina propria. Gastroenterology 1997; 112:1398-403.

17 Vogel G. Can old cells learn new tricks? Science 2000;287:1418

18 Green AJ, Sepp T, Yates JRW. Clonality of tuberous sclerosis hamartomas shown by non-random X-chromosome inactivation. Hum Genet 1996;97: $240-3$

19 Abeln ECA, Smit VT, Wessels JW, et al. Molecular genetic evidence for the conversion hypothesis of the origin of malignant mixed mullerian tumours. f Pathol 1997;183:424-31. 